\title{
¿El Ginkgo Biloba mejora los síntomas cognitivos y el funcionamiento social en pacientes con demencia?
}

A placebo-controlled, double-blind, randomized trial of an extract of Ginkgo Biloba for dementia

Pierre L. Le bars, Martin Katz, Nancy Berman, et al. JAMA 1997; 278:1327-1332

\section{Objetivo}

Evaluar la eficacia y seguridad del uso de extracto de Ginkgo Biloba (GB) en pacientes con enfermedad de Alzheimer (ALZ) y demencia multi-infarto (MI)

\section{Diseño}

Ensayo controlado, doble ciego, randomizado, multicéntrico, de grupos paralelos. Seguimiento: 52 semanas.

Lugar

Seis centros de EE.UU

\section{Pacientes}

Se incluyeron 327 pacientes de ambos sexos mayores de 45 años, con demencia según criterios del DSM III R e ICD-10, tanto ALZ y MI; estadio leve a moderadamente severo. Minimental Test $=9$ a 26 y GDS (Escala de Deterioro Global $)=3$ a 6 . Se excluyeron pacientes con enfermedades clínicas significativas, psiquiátricas y/o neurológicas concomitantes.

\section{Intervención}

GB $120 \mathrm{mg} /$ día $(\mathrm{n}=166)$ o a placebo $(\mathrm{n}=161)$.

\section{Medición de los resultados principales}

Se valoraron los cambios en las siguientes áreas: 1) Deterioro Cognitivo con la subescala para la evaluación de trastornos cognitivos de la ADAS-Cog (Escala de Valoración de Enfermedad de Alzheimer); 2) Actividades de la vida diaria y comportamiento social con la GERRI (Escala de Evaluación Geriátrica realizada por Familiares o Cuidadores; 3) Cambios Psicopatológicos Generales con la CGIC (Imprešión del Médico del Cambio Global).

\section{Resultados principales}

Del análisis por intención de tratar* de 309 pacientes, 202 pudieron evaluarse a las 52 semanas. El grupo GB presentó menor deterioro cognitivo (1.4 puntos mejor en ADAS-Cog, $\mathrm{p}=0.04$ ) y en la escala GERRI ( 0.14 puntos mejor; $\mathrm{p}<0.004)$ que el grupo placebo. No se encontraron diferencias con el CGIC ( $\mathrm{p}=0.77$ ) Para el análisis de eficacia, se encontraron diferencias estadísticamente significativas entre ambos grupos, ADAS-Cog $(\mathrm{p}=0.005)$ y GERRI ( $\mathrm{p}=0.003$ ). De los 309 pacientes incluídos inicialmente en el análisis por intención de tratar, solamente el $50 \%$ del grupo GB (78/155) y el $38 \%$ del grupo placebo (59/154) completaron el estudio hasta la semana 52. Los datos perdidos fueron reemplazados por los obtenidos luego de por lo menos 20 semanas de tratamiento. No se encontraron diferencias estadísticamente significativas entre ambos grupos, con respecto a la incidencia y severidad de efectos adversos. Un análisis por separado fue realizado con el subgrupo de pacientes con diagnóstico de Enfermedad de ALZ ( $n=236)$ en el cual se observó un patrón similar en los resultados.

\section{Conclusiones}

La utilización de GB en pacientes con demencia, fue segura y capaz de estabilizar el curso de la enfermedad, además de mejorar los síntomas cognitivos y la funcionalidad en la vida diaria.

Fuente de financiamiento: Dr. Willmar Schwabe Pharmaceuticals.

\section{Comentario}

El uso de Ginkgo Biloba en el tratamiento de síndromes demenciales, se basa en resultados positivos de unos pocos ensayos clínicos controlados europeos, que en su mayoría no incluían la valoración de trastornos cognitivos y de conducta. Este trabajo sugiere la eficacia del uso de GB en pacientes con diagnóstico de demencia. Los cambios en el grupo GB fueron objetivamente medidos por ADAS-Cog, y fueron reconocidos por los cuidadores y familiares a través del GERRI. Por el contrario, al no haber sido detectados por la impresión de los médicos (CGIC), queda planteado el interrogante si el efecto observado en el grupo tratamiento fue de la suficiente magnitud como para producir manifestaciones clínicas detectables. Otras limitaciones de este trabajo son:

1) No evalúa el tratamiento a largo plazo, los efectos por supresión de la droga o el tratamiento con diferentes dosis (1);2) El promedio de años de educación de 14, no es comparable con el de nuestra población anciana; 3 ) Debido al pequeño número de pacientes, no se realizó un análisis del grupo con demencia multiinfarto, ni tampoco fueron analizados los diferentes grupos según grado de severidad de la enfermedad. No queda claro entonces cómo y cuánto influyen las características clínicas de la población (diagnóstico y estadificación), en la respuesta al tratamiento; 4)
Aunque el estudio plantea el análisis por intención de tratar*, los datos reportados como evaluables corresponden a 202 pacientes ( 97 GB y 105 placebo) en lugar de los 309 inicialmente randomizados. Más aún, el hecho de considerar como válidos datos obtenidos tempranamente en el estudio (semana 20) y no proveer información sobre el gran número de pacientes que abandonaron antes de la semana 52, puede modificar el resultado final subestimando o sobrestimando el efecto de la intervención. Por lo tanto, si bien el estudio respalda el uso de GB en pacientes con demencia, deben considerarse las limitaciones expuestas al evaluar esta recomendación.

* Ver glosario

Dra. Cinthya Marturano Servicio de Clínica Médica Hospital Italiano de Buenos Aires

Referencias

1.Kanowski S.Herrman W, et Al. Proof of efficacy of the Ginkgo Biloba special extract to moderate primary degenerative dementia of the Alzhei mer type or Multi Infart Dementia. Pharmacopsychiatry.1996;29:47-56. 